\title{
Improving the Sensitivity of Research Publication Indices Using Exponential Function and the Area under Curve
}

\author{
Majdi A. Maabreh ${ }^{1}$, Izzat M. Alsmadi ${ }^{2}$ and Mohammed N. Al-Kabi ${ }^{3}$ \\ ${ }^{1}$ Computer Center, Hashemite University, P.O. Box 150459, 13115 Zarqa, Jordan \\ ${ }^{2}$ Department of Information Systems, Prince Sultan University, Riyadh, Saudi Arabia \\ ${ }^{3}$ Faculty of Sciences and Information Technology, Zarqa University, 13132 Zarqa, \\ Jordan \\ majdi@hu.edu.jo,ialsmadi@cis.psu.edu.sa,malkabi@zu.edu.jo
}

\begin{abstract}
In collecting and analyzing information about recent research papers, authors, and journals with their citation and indices, we noticed that in many cases such indices fail to reflect reality and are incapable to distinguish between different authors in some cases while they clearly have different research profiles. There are many research papers that can be found in literature that discussed the behavior and limitations of the current citation or publication indices. Most of them referred the thensitivity problem and the fact that most indices neglect some publications during index calculaton, These two problems are the most important limitations that may judge index fairness. In this paper, we proposed new indices; MIExp-Index using exponential function and MIArea-Index using the concept of the area under curve (AUC) in order to enhance, the sensitivity and thus the fairness of index assessment. To demonstrate our two novel indices, data of real and hypothetical authors were used to demonstrate these (tyo approaches. The results showed that both of the proposed indices are very sensitiveforeach single citation that the paper receives after being published. In addition, the two indices considen all authors' publications, even the new ones, into account.
\end{abstract}

Keywords. H-Index, MIEXPIndex, MIArea-Index, Research assessment, Impact Factor, Citations-based indices.

\section{Introduction}

The need to a criterion to assess the quality of scientific outputs of a scholar (scientist), conference, journal, university, etc. leads to the emergence of a number of performance indicators such as $H$-index, G-index, etc. These performance indicators are classified into three casses according to (Franceschet, 2009); Productivity metrics, impact metrics and hybrld metrics where the number of citations is mainly used to assess the performance indicators of those three classes. These indicators are essential in our present time to make objective judgment about activities such as: Scientific promotion, research grants, recruitment, selection of scientific committees, and awards.

The assessment of research quality was based on one of the bibliometric indicators (e.g. total number of papers, total number of citations) before inventing performance indicators in 2005 by Jorge Hirsch. This means that no formula exists to combine more than one bibliometric indicators. The proposal of the first impact metric (citation index) $H$-index by Argentine American physicist Jorge Hirsch (Hirsch 2005) invokes scientists to propose 
several other performance metrics in general and impact metrics in particular. These metrics are used to evaluate the research impact of a particular: author, paper or journal. Jorge Hirsch metric (H-index) is used to quantify the scientific output of a single researcher by one indicator, where this indicator combines the number of publications and number of citations in one formula. Hirsch indicator $(H$-index) is an objective metric which is easy to compute and verify. $H$-index is considered better than one criterion indicators such as: Total number of citations, rate of citation per paper, or total number of publications. $H$-index receives a positive acceptance by a number of studies which shows its usefulness such as (Bornmann, \& Daniel, 2005). Glänzel (2006b) shows also that one of the $H$-index strengths is its ability to assess a small set of articles. (Cronin, \& Meho, 2006) used the H-index to rank 31 wellknown information science scientists, where the self citation is included and excluded to conclude that self citation has no influential effect on the rank of those scientists. Many authors discussed the pros and cons of H-index. Further, many variants to Hirsch indicator are casted. For example (Bornmann and Daniel, 2007) found that around 30 papers oublished during the first year after publishing $H$-index.

Several researchers realized shortly after publishing Hirsch inđex that it can be used to evaluate also scientific journals, universities, members of national academies, etc. (Braun, Glänzel, and Schubert, 2006). Therefore the scope of using $H$-index is not restricted to quantifying the research outputs of different scientists around the world

Hirsch himself in (2007) argues that his index can be used to predict any scientist's scientific research future productivity, and proves it is better for prediction than other bibliometric indicators. He claims also that $H$-inde $x$ is a better predicator than other indicators even those variants which assign more weight on highly cted articles.

Several researchers criticize the $H$-index early since it was released. (Glänzel, 2006a) lists 8 main disadvantages of $H$-index. (Jin, Diang, Rousseat and Egghe, 2007) also presents 14 pros and cons of $H$-index in their study. (Hirsch 2005) noted that the scientific research field may affect the $H$-index value, therefore the highest $H$-index value of a physicist (namely, Witten E) was 110, and the highes H-index value of a scientist (namely, Snyder SH) was 192 where difference between both is significant, Egghe (2006a) noticed early that once a paper belongs to the $h$-defined inner class ( known as $h$-core), it becomes ineffective whether it has 0 or 1000 extra citations, and $\mathrm{H}$-index should be then restricted to compare scientist's with equal years of expenenca Egghe (2006a) also noticed that $H$-index value is a non-decreasing one. As a result, an inactive scientist (later on his/her life) will maintain the same $H$-index value. Accordingly, the study (Egghe 2006b) proposed one of the most important variants of the $\mathrm{H}$ index which is called G-index. The justification of proposing this new variant ( $G$-index) was due to insensitiveness of the $H$-index to a set of non-cited (or modest cited) papers and to a set of highly cited papers. (Egghe, 2006c) shows that $G$-index is sensitive to both cases of low or high number of citations for publications.

Examples of other citation indices that were proposed in relevant research papers in addition to the $H$-index include: $g_{-}, h(2)-, w$-, and $h w$-Index where those depend on $H$-index behavior in calculating the impact value, in addition to: $h g_{-}^{-}, q^{2}-, A R-, m$-quotient, $m-, e-, A-$, $r$ - $, w, j$-index, etc. where those directly depend on $H$-index value in their calculations. This dependency relation is considered as a limitation of the proposed indices as they should calculate the $h$ value before their assessment.

Each index represents a different formula to calculate the impact value based largely on only two factors: Author's number of publications and number of citations for all his/her publications. Some of the proposed indices added new attributes such as: Publications dates or periods, average number of publications, citations, etc. Examples of such indices include: Contemporary $H$-index, $m$-quotient, and $A R$ - index. 
The number of citations is still used as the main value for research assessment in most current indices. In the web, each webpage takes its popularity from those WebPages that point to it regardless of the webpage contents. If the webpage has for example $100 \mathrm{In}$-links, then it can be considered as more popular than another one which has just 5 In-Links. In the research community, the matter is not different. The paper which has 100 citations is more important or influential than another one which has just 5 citations, regardless of the actual research value in both.

All indices, including our proposed one in this paper, do not take into account the contents but just the In-Links or citations. Since the number of citations is the primary value for research indices' calculations then this value should be thoroughly investigated and calculated. However, even if we assume that the citation values are accurately collected, the citation indices do not calculate these values in fairly manners. For example, if we havesa software program that is developed for collecting authors' publications and citations, and the software returns information about an author who has just 5 publications $\left(P_{1}, P_{2}, P_{3}, P_{4}\right.$, and $\left.P_{5}\right)$. Further, these publications have citation values of: $\{100,50,20,10$, and 5$\}$ respectively. According to the H-index citation point of view, the author has an H-index value of 5 . However, the H-index value will not change, if we knew-that the software while collecting papers' citations missed many citations for the authors 'publications. Let's assume that the correct citation values are: $\{500,250,200,10$, and 9$\}$. H-index in this case will also stay the same as earlier. This means that the $H$-index did not consider how many citations the paper has as far as the citation value exceeds its order value $(i . e .1,2,3,4$, etc.). If we assume that the software also misses some publications for the same author, for example $\left\{P_{6}, P_{7}\right.$, and $\left.P_{8}\right\}$, and these papers have citation values of. $\{2,2,1\}$ gespectively, these possibly recent publications will not change the $H$-index value. This insensitivity of the index to variations of a single author publications and citations or variations between different authors is what triggered our trial to propose more sensitive indices.

In this paper, new citation indices are proposed and new mathematical formulas are applied in order to tackle some of the issues mentioned above. We will benefit from the sensitivity of the exponential function as well as the concept of the area under curve in developing more sensitive indices which take all citation into consideration.

\section{Related Work}

$H$-index with its simplicity built a baseline for most of later publications indices. All $\mathrm{H}$ index variants tried to solve one or more limitation of the H-index. For example, (Schreiber, Malesios, Psarakis, 2012) study presented $17 \mathrm{H}$-index variants and examined the performance and properties of each one of them. (Bornmann, Mutz, Hug, \& Daniel 2011) conducted a larger study than the previous one and presented in their study the computation of correlations between the H-index and 37 of its variants. Authors found that the H-index is highly correlated With most of its variants. The high correlation between $H$-index and most of its variants means that these variants are somewhat redundant to $H$-index. (SCI2S, 2013) presented and classified a large number of indices based on $H$-index.

One of the disadvantages of $H$-index is in its neglecting of the number of authors in research papers and also their order in the paper. Therefore a complementary version of $\mathrm{H}$ index called $h_{I}$ is presented by (Batista, Campiteli, \& Kinouchi, 2006) to solve this problem based on conclusions of previous studies showing that the number of authors generally influenced the number of citations. If all studies of an author are solo for the author as a single author then the $H$-index value is equal to $h_{I}$-index value. In addition, $h_{I}$-index values are generally less sensitive to the scientific research field relative to $H$-index values. Schreiber (2008) presented a solution to the number of authors issue by introducing a modified $H$-index 
( $h_{m}$-index). Author compares the performance of $H$-index, $h_{I}$-index, and $h_{m}$-index using the scientific outputs of eight famous physicists, where author claims that $h_{m}$-index was a better representative for author's research production.

Egghe (2006b) proposed a new index called $G$-index. G-index represents an improvement to $H$-index, since it takes into its account highly cited papers, which are ignored by $H$-index. Nonetheless, $G$-index could not solve all the problems observed in $H$-index. For example both $H$-index and $G$-index are insensitive to the number of coauthors of a given article. To solve this problem $h_{r}$-index and $h_{m}$-index were introduced by (Batista, Campiteli and Kinouchi, 2006) and Schreiber (2008) as mentioned before. (Schreiber, 2009), Schreiber (2010a) and Schreiber $(2010 b)$ present a modified $G$-index $\left(g_{m}\right.$-index) which takes into account the number of coauthors in each article. Therefore the values of $g_{m}$-index of different scientists who have multi-author articles will be lower than the values of G-index for the same scientists. This means that $g_{m}$-index will be in favor of scientists who have fewer pumbers of authors.

One of the H-index issues is the precision problem. This occurs in some cases when an author publishes papers under different names as noticed by (Jin et a1., 2007). Different names are due to misspellings, transliterated names, marriage, and changing names. Kosmulski (2006) $h(2)$-index is easier to compute than H-index and is defined as the highest number $h(2)$ of articles that have at least $\left((h(2))^{2}\right.$ or more citations. $h(2)$-index aims to solve the problem of insensitivity of the $H$-index to highly cited articles. Kosmulski (2006) defines the scientist's $h(2)$-index as "the highest natural number such that $h(2)$ most-cited papers receive each at least $[h(2)]^{2}$ citations." $h(2)$-index helps to reduce the precision problem according to (Jin et al., 2007). Wu (2010) proposes a novel performance index called $W$ index. It is also more sensitive than $H$-index for highty cited articles.

Jin (2006) and Rousseau (2006) discussed an $A$-index to measure citation intensity. The $A$ index is defined as the average number of citations received by the articles given in the $h$ core. Rousseau (2006) called it $A$-index since it is related to average values. It is argued that the $A$-index can solve the problem of highy cited articles ignored by $H$-index. This makes $A$ index close to the $G$-index, since it also solves the same problem. One of the problems facing the $A$-index is summanized by the possibility that a decrease in the value of $A$-index may occur when the number of citations is mcreased since formula involves a division by $H$-index value (Maabreh and Alsmadi, 2012)

Three different indices are presented by (Sidiropoulos, Katsaros, Manolopoulos, 2007), which are variants of $H$-index and called: normalized-, contemporary $H$-index and trend $H$ indices. The comparisen of different scientists according to their publications faces the challenge that those authors may have significantly different profiles in terms of number of publications. As such, Normalized $H$-index is proposed to solve this problem. Trend $H$-index takes into it account citations' age, so that articles cited for few years get lower values than those cite for a long period of time. Contemporary $H$-index takes into its account the age of the article.

Bormmann, Mutz and Daniel, 2008) paper proposes the $m$-index as a variation of the $A$ index M-index is the median number of citations received by papers in the $h$-core. Median is one of the statistical central tendency measures. The number of citations received by a paper in the $h$-core is sorted in descending order to select the middle value easily. M-index is a simple computed metric and needs precedent determination of $\mathrm{H}$-index. Citation distribution counts is usually skewed and is not included in M-index (Bornmann, Mutz, \& Daniel, 2008). An increment in the $h$-core citations may not affect the value of $M$-index helps to reduce the impact of heavily cited papers in the $h$-core. Values of $M$-index are higher than their $H$-index counterparts. 
As mentioned before the $H$-index value is a non-decreasing one and it is insensitive to the age of the paper and actual number of citations. Therefore another complementary version of $H$-index called $A R$-index was introduced by Jin (2007) to solve these limitations. The $A R$ index formula is characterized by its simplicity, since it is equal to "the square root of the sum of the average numbers of citations per year of articles included in the $h$-core". So the values of $A R$-index can decrease according to its formula.

(Jin, Liang, Rousseau and Egghe, 2007) proposed a novel metric to measure the citation intensity in the $h$-core called $R$-index. $R$-index is equal to the square root of the sum of all citations received by articles included in the $h$-core at time $T$. The $R$-index must be used in conjunction with the H-index.

(Egghe, and Rousseau, 2008) paper proposes a citation weighted $H$-index which is called $h_{w}$-index. This performance indicator aims to solve the problem of the $H$-index insensitivity to performance changes similar to the $A R$-index. $h_{w}$-index is equal to the square root of the total number of citation $(s)$ received by the highest number of articles that each pecei ed $s / h$ or more citations. However, similar to $A$-index issue, an increase in the number of citations could lead to a decrease in the value of $h_{w}$-index.

(Alonso, Cabrerizo, Herrera-Viedma and Herrera 2010) paper presents another index called hg-index. It is called so since it is based on both $H$-index and $G$-index as $1 t$ tried to combine both indices into one. The main goal of $h g$-index is to maintain the pros of these two indices (H-index and $G$-index) and minimize their cons. Empirical tests of hg-index by authors show its advantages on both original citations.

(Cabrerizo, Alonso, Herrera-Viedma and Herrera, 2010) paper presents a metric called $q^{2}$ index. $q^{2}$-index includes two dimensions of scientist? scientific research output; First dimension represents the number of articles, and the second dimension represents the number of citations (impact of papers). Authors stated that: $q$-index is based on the geometric mean of $H$-index and the median number of citations received by papers in the $h$-core, i.e., the $M$ index, which allows us to combine the advantages of both indices."

\section{Citations Indices \\ 3.1. Sensitivity and Comprehensiveness Issues}

By sensitivity problem we mean that most citations focus on the $h$-core or the publications that get the largest number of citations. For most citation indices, activities outside the $h$-core are ignored. For example, an author may get 30 new publications in the current year without impacting or changing bis/her citation index value. Furthermore, activities in the $h$-core may not also impact the citation index. Citation indices are largely interested in the publications that are about to pass the edge from the $h$-tail to the $h$-core zone. The $H$-index value would not increase in response to citation increments gained by $h$-core publications. For example if an author has 5 publications that were cited as: $(10,6,3,3,1)$ then author will have an $H$ index alye of 3 . If the publications in $h$-core (i.e. 10, 6, 3) gain more citations in next years, say $(20,15,9)$, and no citations occur in the rest of publications then the H-index will not be changed.

To overcome the $H$-index limitations (Egghe, 2006) proposed the $G$-index, which is now a major index in addition to the H-index. G-index was proposed based on the behavior of $H$ index with some enhancements to improve its sensitivity. However, the " $h$-core" or "big-hits" problem still affect or decide the $G$-index calculations. The $G$-index partially overcomes the sensitivity problem in comparison with the H-index. However, the sensitivity problem can still be seen in the $G$-index with many scenarios. For example, if an author has 5 publications with citation numbers as: $(7,5,4,2$, and 0$)$, then he/she has a $G$-index value of 4 . There will 
be no change on $G$-index value if the author papers' citations became as: $(10,7,4,2$, and 0$)$. The sensitivity problem was also raised with many of $h$ type indices such as: $W-, h(2)-, M-$, $h g-, m$-quotient, $q^{2}, h w$ - and $j$-indices. Another problem was seen through experiments with $M$-index, where the increase in the number of citations has no effect on the $M$-index value, if this increment would not lead to a median change. This problem is also clear in those indices which depend on square root function such as: $A-, R-, A R-, E-, h g_{-}, q^{2}$-indices as examples.

In addition to the sensitivity problem, for what is called $h$-tail (Ye and Rousseau 2009) (i.e. the rest of the publications with the exclusion of $h$-core publications), there is no logical base to exclude those publications from $H$-index calculations. While, it makes sense to give more weight to publications with the high number of citations, however, this should not mean to, almost, totally ignore the rest of publications. This problem was also raised in all indices which depend on $h$-core contents such as: $A$-, $R$-, $A R-, E-, M-$, and $h g$. In many scenarios, the rest of publications may have more than 10 citations and may be neglected.

In terms of comprehensiveness, we discussed some of the earlier issues related to the limitations of citation indices and ignoring some publications, citations. Some other important information such as the number or sequence of authors, age of paper, etc. can be also categorized under incomprehensiveness issues.

\subsection{MIExponential (MIExp-Index) Using the Exponential Function}

Most of the indices presented in this study use the number of citations received by the articles to quantify the value of scientist's publications. Typically, high citation to an article should indicate useful information in the papers for others to cite from or refer to.

The number of citations received by the article varies from zero to thousands. We have to distinguish between the real number of citations (zero or positive integer) and the order of these values as in $H$-index. Using the number of citations could be impractical without preprocessing or normalizations.

Most of research studies can be cited from the date of its publication, and some studies are cited before its date of publication (e.g. An pre-processing or publication cases). The number of citations for each paper is accumulated through years. Suppose a paper $X$ was published in 1990 and from its date of publicationand till now each year the total number of citations of paper $X$ is increased. It the present number of citations of paper $X$ is 1000 . This number was the lowest in the date of publication and currently is the maximum. In other ways, total number of citations should always increase, or stay fixed with time and not decrease. That means when the age of any paper is increased, so the number of citations for this paper should not be decreased.

A common loganithm with respect to the base $b=10$ is used in our proposed formula to deal with the cumulative nature of the number of citations. Therefore, the number of citations is transformed-into a smaller number. From a mathematical point of view logarithms can be used to find the growing rate of the individual authors' research paper during their academic age. Logarithms can also help to answer the following question: How can we model the cilation growth in a given time interval from $X$ level to $X+n$ level? We will add number 1 to the citation values in order to deal with those authors who have publications with zero citations since ( $\log _{10} 0$ is undefined).

To get a sensitive formula to any change in the number of citations, a natural exponential function is used in our proposed formula (1), where $e$ is used as a base for our proposed function. A common logarithm to base 10 of the number of citation plus 1 represents the exponent of our new proposed formula. The Exponential function is considered as a sensitive function in response to any change in the input values. 
Then,

$$
\text { MIExp } \quad-\text { Index }=e^{\log _{10}(\text { No. of citations }+1)}
$$

\subsection{MIArea (MIArea-Index) Using the Concept of the Area under Curve}

The area under curve is a mathematical concept that can be used in many applications. In this study, we used this concept to represent authors' publications and their citation values. The $y$-axis corresponds to the number of citations received by a paper, whereas the $x$-axis represents the number of authors' publications. For example, Table 1 represents such authors' research papers.

Table 1. A sample of author research papers

\begin{tabular}{lllllllllll|l|l}
\hline $\begin{array}{l}\text { No. of } \\
\text { Publications }\end{array}$ & 1 & 2 & 3 & 4 & 5 & 6 & 7 & 8 & 9 & 10 \\
\hline $\begin{array}{l}\text { No. of } \\
\text { Citations }\end{array}$ & 12 & 7 & 5 & 4 & 4 & 3 & 3 & & & 1 & 1 \\
\hline
\end{tabular}

The citation curve exhibited in Figure 1 was based on the citations presented in Table 1 . The citation curve shows clearly the drop out in the number of citations

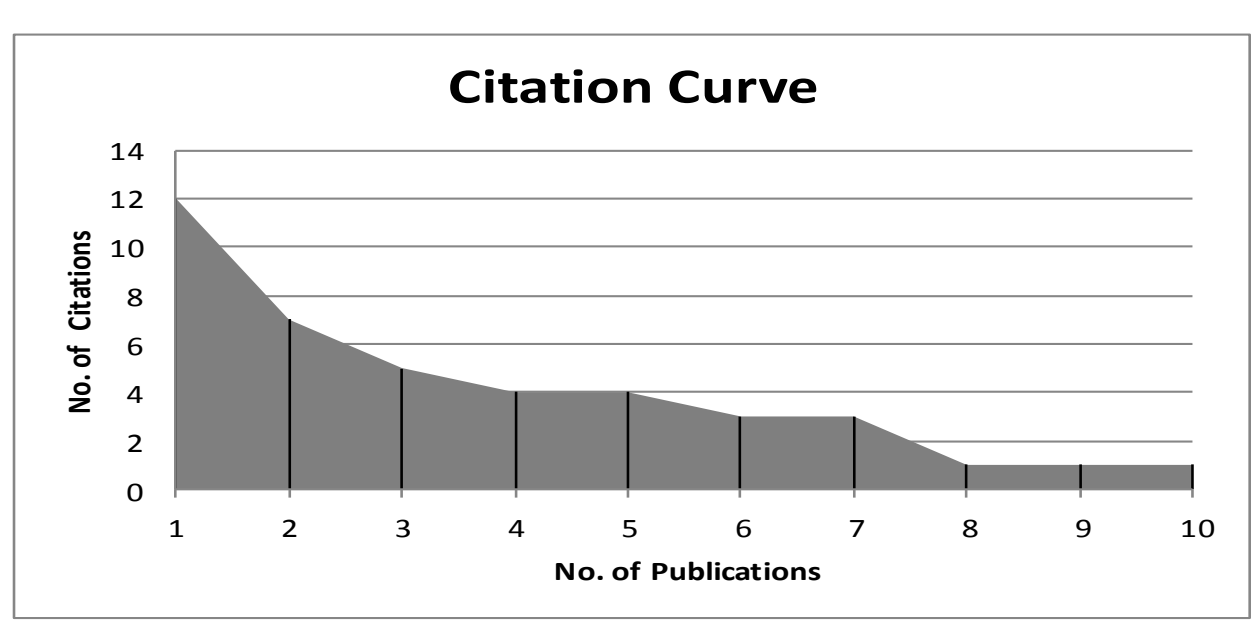

Figure 1. Citations vs. Publications

We may use two methods to find the area under curve or the area between the curve and the $x$-axis. First we can use a definite integral to compute area under a curve. However this method needs the presence of a function which generates $y$-values which can be computationally complex. In fact, there is no clear, formal or general relationship between number of publications and number of citations. Here, we assume that papers for each author with their citations can form an area chart like those areas presented in Figure 1. The second method needs not any knowledge in calculus as MS Excel trend line option can be a simple solution.

In Figure 1, each publication and its citations form a sub-area of the whole chart. Furthermore, each sub-area is a trapezoid or a special type of trapezoid. As such, to estimate the area of a trapezoid, the following formula is used:

$$
\frac{b-a}{2 N}\left(f\left(x_{1}\right)+2 f\left(x_{2}\right)+2 f\left(x_{3}\right)+\ldots 2 f\left(x_{N}\right)+2 f\left(x_{N+1}\right)\right)
$$


Where, the value of $b$ represents the order of the author latest publication(s) $(1,2,3, n)$. The value of $a$ in this approach is always equal to $1 . N$ refers to the number of generated trapezoids, and it equals to $(b-a) . f\left(x_{i}\right)$ refers to the number of citations that the paper $(i)$ received, where $i=1,2,3, \ldots, n$.

\section{Conditions:}

Using the Area under Curve (AUC) discussed above is regulated by the following conditions:

1- It is applicable only for those authors who have more than one publication. The author who has one publication will get ZERO area units even if the single publication gained more than 100 citations. The value Zero means then that the author has no or single publication.

2- The citation values should follow the $H$-index order (i.e. descending order)

3- The publications with zero citation will be ignored as there is no specific impact yet.

For the above example shown in Figure 1, the values are ordered as follows: $(12,7,5,4,4,3$, $3,1,1$, and 1 ).

$$
\begin{aligned}
A U C & =\frac{10-1}{2 \times 9}(12+(2 \times 7)+(2 \times 5)+(2 \times 4)+(2 \times 4)+(2 \times 3)+(2 \times 3)+(2 \times 1)+(2 \times 1)+1) \\
& =34.5 \text { Area Unit }
\end{aligned}
$$

If we assume that any paper of this author gains 1 more citation value, then the AUC will be 39 Area Unit. Therefore, the MIArea-index (Which equals to AUC) is very sensitive for citation values. Moreover, the publieation will not be neglected even if it has at least one citation. For normalization issue we can usê a factor such as $\alpha$, where $\alpha$ value may be $=\{0.1$, $0.2,0.25 \ldots .0 .5 \ldots 1\}$. Identifying the value of $\alpha$ needs further studies and extensive experiments. In this study we assume that $\alpha=1$.

\subsection{Example}

An example showing the calculations of the two proposed novel indices (MIExp- and MIArea - Index) is presented this section. Two authors were used in this example, where each one of these two aythors has 10 research papers, which differently cited as shown in Table 2.

Table 2 Two authors scientific outputs with their citations

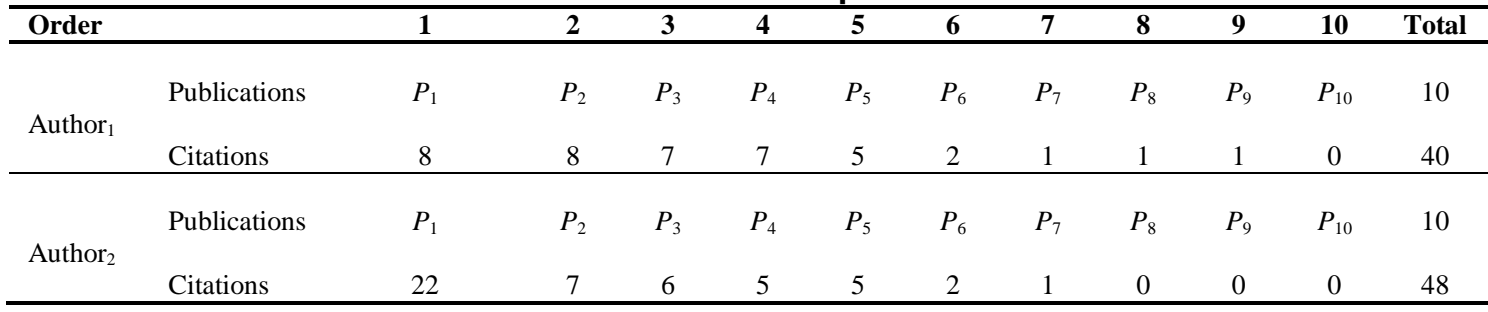

The calculations of MIExp-Index are presented first in this section using the data presented in Table 2.

MIExp-Index for the first author (Author ${ }_{1}$ ) equals to:

$$
\text { MIExp - Index }=e^{\log _{10}(40+1)}=5.017
$$

MIExp-Index for the second author (Author ${ }_{2}$ ) equals to:

$$
\text { MIExp - Index }=e^{\log _{10}(48+1)}=5.421
$$


Next the calculations of MIArea-Index are presented using the data presented in Table 2.

MIArea-Index for the first author (Author ${ }_{1}$ ) equals to:

$$
\begin{aligned}
& \text { MIArea }- \text { Index }=\left(\frac{10-1}{2 \times 9}\right)\left(\begin{array}{l}
8+(2 \times 8)+(2 \times 7)+(2 \times 7)+(2 \times 5) \\
+(2 \times 2)+(2 \times 1)+(2 \times 1)+1
\end{array}\right) \\
& \text { MIArea - Index }=35.5 \text { Area unit. }
\end{aligned}
$$

MIArea-Index for the second author (Author 2 ) equals to:

$$
\begin{aligned}
& \text { MIArea }- \text { Index }=\left(\frac{10-1}{2 \times 9}\right)\left(\begin{array}{l}
22+(2 \times 7)+(2 \times 6)+(2 \times 5)+(2 \times 5) \\
+(2 \times 2)+1
\end{array}\right) \\
& \text { MIArea }- \text { Index }=36.5 \text { Area unit. }
\end{aligned}
$$

\section{Evaluation}

In order to assess the proposed two indices, and show how they may orereome some of $H$ and G-index weaknesses; mainly, the "Sensitivity" and "Comprehensiveness" problems, we will conduct two simple but significant experiments. In the first experiment, we will use artificial examples of authors, publications and citations. In the second one, we will show how the proposed indices behave with real data in-comparison with $H$-and $G$-indices.

\subsection{First Experiment}

The assessment process is conducted using four different scenarios listed in Table 3. We believe that these scenarios simulate realistie scenarios of researchers.

The first scenario (FF) includes those authors who can be classified as newcomers or beginners, where the publication volume and ts influence are somewhat small. The second scenario (FM) represents those authors who are usually interested in producing few core papers, large influence, regardless of the production volume. The authors in scenario three (MF) focus on volume or number of publications with possibly few number of citations, at least at the beginning. For the last one (MM) represents senior authors with large number of publications and citations. We can say that in any research field researchers may fall largely in one of those four classes orcategories.

Table 3. First Experiment of Four Simulated Cases of Researchers

\begin{tabular}{lll}
\hline Scenario ID & \# of Publications & \# of Citations \\
\hline $\mathbf{1}$ & Few & Few \\
$\mathbf{2}$ & Few & Many \\
$\mathbf{3}$ & Many & Few \\
$\mathbf{4}$ & Many & Many \\
\hline
\end{tabular}

For each scenario, we will simulate the increase in citations value during the publications life time by applying three of common citation increments. Table 4 shows the increment scenaros. We use these scenarios in order to examine the sensitivity of the two indices under study in dealing with citation increments with different scenarios of publication and citation values.

Table 4.Citation Increments

\begin{tabular}{cl}
\hline Scenario-ID & Description \\
\hline $\mathbf{1}$ & Add citations to the first publication where they are in a \\
$\mathbf{2}$ & descending order. \\
$\mathbf{3}$ & Add citations to others except the first one, where they are in a \\
descending order.
\end{tabular}




\subsubsection{The First Suggested Scenario}

This scenario simulates new researchers who have few numbers of publications and their publications have a few numbers of citations. Table 5 shows four authors who are classified as new researchers.

Table 5. New Researchers

\begin{tabular}{|c|c|c|c|c|c|c|c|c|c|c|c|}
\hline \multirow{2}{*}{ Author $_{1}$} & Pub. 1 & 2 & 3 & 4 & \multirow{2}{*}{ Author $_{2}$} & Pub. & 1 & 2 & 3 & 4 & 5 \\
\hline & Cit. & 1 & 0 & 0 & & Cit. & 3 & 2 & 1 & 1 & 0 \\
\hline \multirow{2}{*}{ Author $_{3}$} & Pub. & 2 & 3 & X & \multirow{2}{*}{ Author $_{4}$} & Pub. & 1 & 2 & 3 & X & X \\
\hline & Cit. & 1 & 0 & X & & Cit. & 8 & 4 & 0 & $X$ & X \\
\hline
\end{tabular}

The index values of these four cases are presented in table 6 .

Table 6. MIExp and MIArea values for the four new researchers

\begin{tabular}{lcccccc}
\hline & $\begin{array}{c}\text { Total } \\
\text { Publications }\end{array}$ & $\begin{array}{c}\text { Total } \\
\text { Citations }\end{array}$ & H-index & G-index & MIExp-Index & MIArea Index \\
\hline Author & 4 & 3 & 1 & 1 & 1.826 & 1.5 \\
Author $_{2}$ & 5 & 7 & 2 & 2 & 2.467 & 5 \\
Author $_{3}$ & 3 & 4 & 1 & 2 & 2 & 6 \\
Author $_{4}$ & 3 & 12 & 2 & 2 & 3.046 & \\
\hline
\end{tabular}

Table 6 shows a considerable difference in MIExp and MIArea-index values for these four authors.

MIExp and MIArea measure the impact of authors (Author 1 and Author ${ }_{3}$ ), and (Author 2 and Author $_{4}$ ) in different aspects in comparisen with $h$ values that were almost the same for the four cases. Variations can be also noticed between proposed indices and $G$-index values. Even though the assessments are somerbat close to each other, but the comparison between Author $_{2}$ and Author $_{3}$ with respect to values of $G$-index and the proposed indices may indicate that they are not consistent.

\subsubsection{The Second Suggested Scenario}

In this scenario, we concentrate on those authors who have few publications and many citations. These prolications can be classified as core papers in their field. Table 7 shows artificial examples of this scenario.

There are no differences between the $H$-index values shown in table 7 . They have the same $h$-level (i.e. 10). In table 7 the total number of citations of author A23 is 8,444 and the total number of citations of athor A24 is 34,033. The difference between these two values is large $(25,589)$, and the H-index value determines the most influential author. In this case the number of papers is ignored. The G-index and our two proposed indices (MIExp and MIArea) show different citation values for the same scenario.

After applying the first increment scenario, the $H$-index and the $G$-index did not consider the newalues of citations into account. On the other hand, the MIExp-index and MIAreaindervalues are affected by citation changes. Both of MIExp-index and MIArea-index are sensitive to citation changes.

There are no criteria to increase the values of citations in the second scenario. The results show that both of $H$ - and $G$-indices' values have not changed, while the MIExp and MIArea values were impacted by the new citations. 
Table 7. Highly Cited Authors with Few Publications

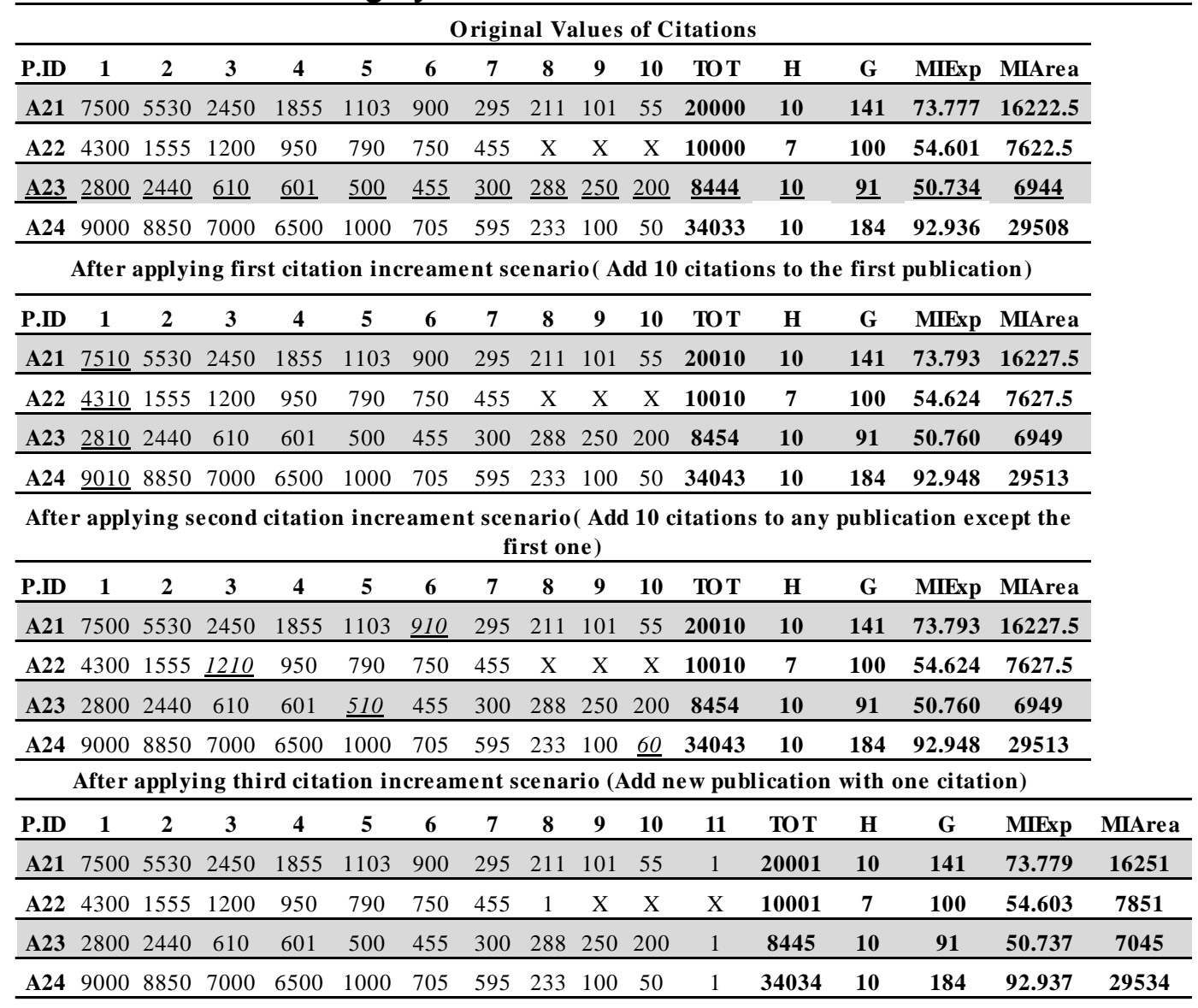

The third increment case shown in table 8 shows how the new two proposed indices (MIExp and MIArea) bebave with the extension of the publication list. Values of MIExp and MIArea indices are alfected and changed when one publication with one citation is added. On the other hand, the index values of $H$ - and $G$-indices have not changes. In Table 8 the values of MIArea-index of scenario 3 are completely different from the values of MIArea-index of scenario 1, where the yalues of MIArea-index of scenario 3 are higher than the values of MIArea-index of seenarlo 1 using the trapezoidal rule to compute those values which represent area under the curve. In addition, these values can be used to predict the number of citations for different articles published by the same author. Table 8 shows the second scenario with the three increment cases. The results show that the proposed indices (MIExp and MIArea) are more sensitive in response to the changes in the total number of citations if those indices were compared with traditional $H$ - and $G$-indices.

\subsubsection{The Third Suggested Scenario}

Table 8 shows the three cases and the results of the third suggested scenario of publications and citation values, and the effect of the three increment cases on the evaluated indices. As shown in Table 8, the G-index was slightly affected in response to the citation changes in comparison with $H$-index where the citation increment values made no impact on the $H$-index. However, the sensitivity degree of our proposed indices (MIExp and MIArea) is noticeable in response to the increment in the number of citations and publications presented in Table 8 . 


\subsubsection{The Fourth Suggested Scenario}

Table 9 shows artificial examples of publications, citations and the behavior of the indices understudy. The 100 new citations added to the number of citations in the first and second increment cases have no effect on the values of $H$ - and $G$ - indices. Table 9 shows also clearly the sensitivity of the new proposed indices (MIExp and MIArea) to those new 100 citations added to the original citations. Adding one new publication with one citation to the current publication list for each author affects also the values of MIExp and MIArea indices. Therefore adding one citation to thousands of citations for a long list of publications can positively affect the values of proposed indices whereas it has no effect on the values of $\mathrm{H}$ and $G$-indices.

Table 8. Cases of a large number of publications and low citation

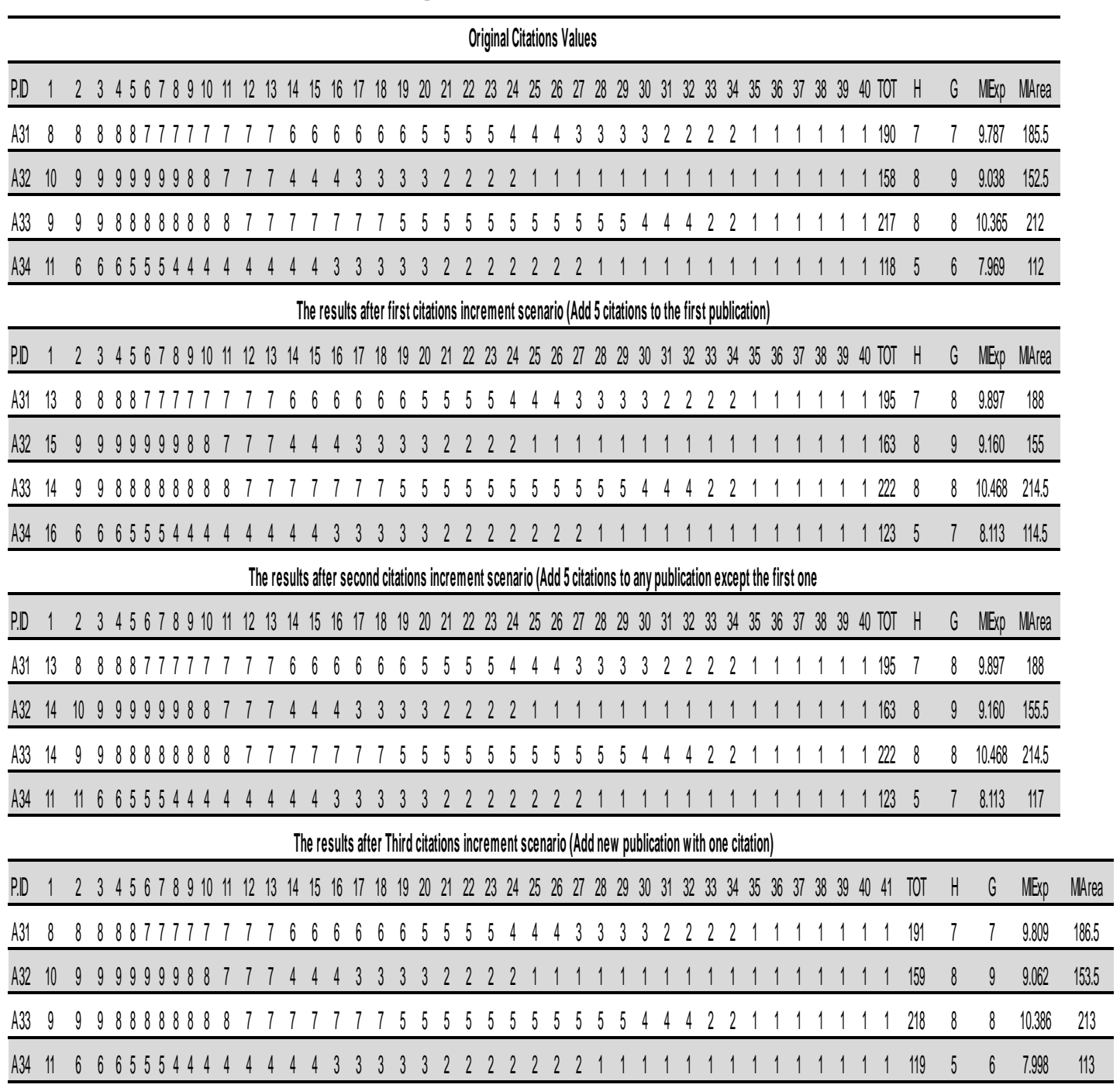


Table 9. Cases of many publications and citations

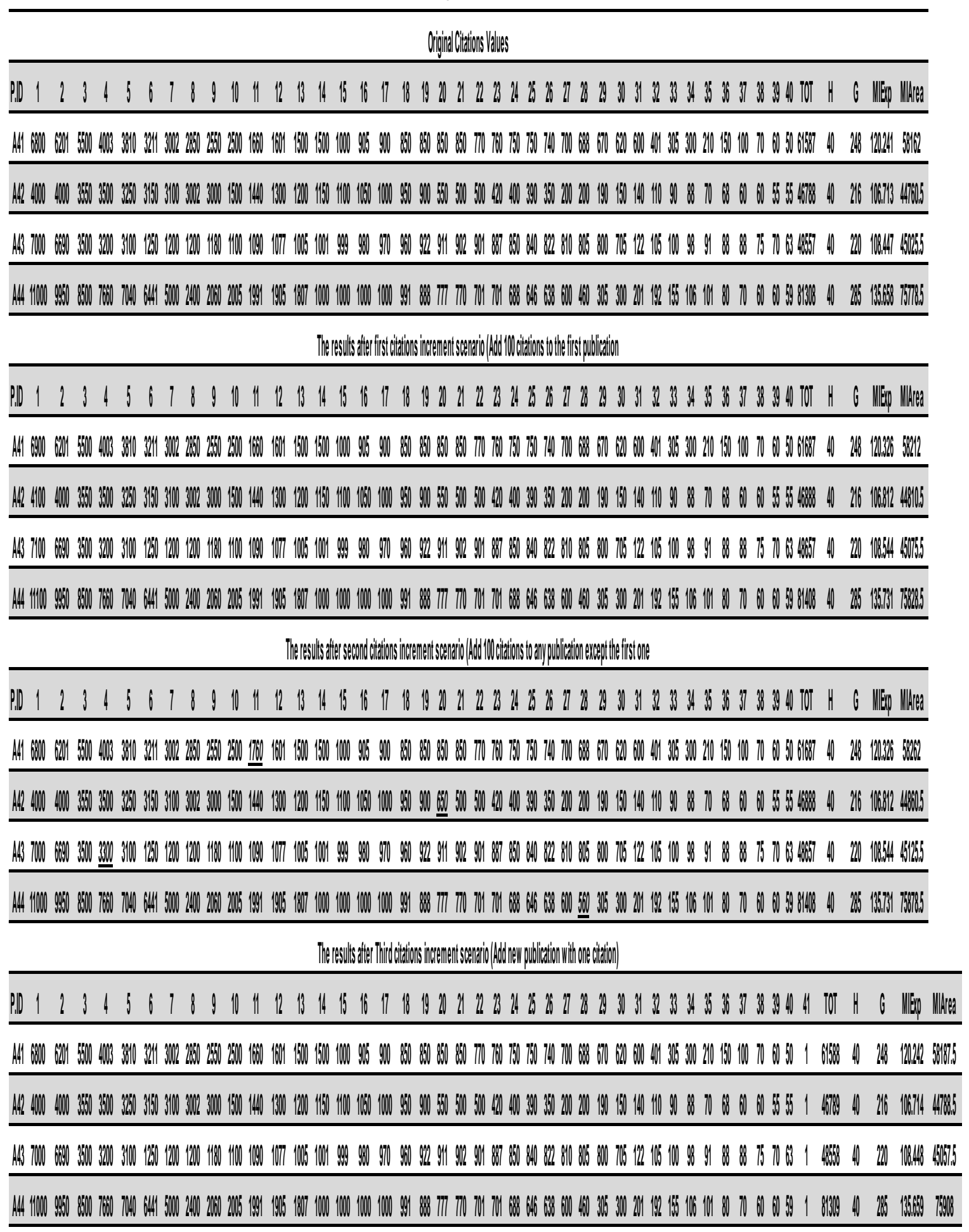




\subsection{Applying MIExp and MIArea indices on real data}

Table 10 shows the first 15 authors in the field of software engineering ordered as they appear in the website of Microsoft academic research (http://academic.research.microsoft.com). Microsoft academic uses special criteria to rank the authors. Their ranking policy depends mainly on H-index and other factors such as: distribution of publications and citations of author works. We applied and examined the four indices under study on these top ranked authors. The $G$-index behaves in a more fairly manner in comparison with $H$-index to assess the authors' publications. For example, the use of $G$ index shows that the studies of Edmund Clarke are more influential than the studies of Victor Basili. It is clear that the use of $\mathrm{H}$-index in such situations leads to unfair results, since it is strange for the index to rank the author with: 453 publications and 45,375 total citations of Edmund Clarke in a rank after an author with: 603 publications and 20,367 total citations of Victor Basili.

Table 10. Microsoft Academic - Top 15 Researchers in Software Engineering

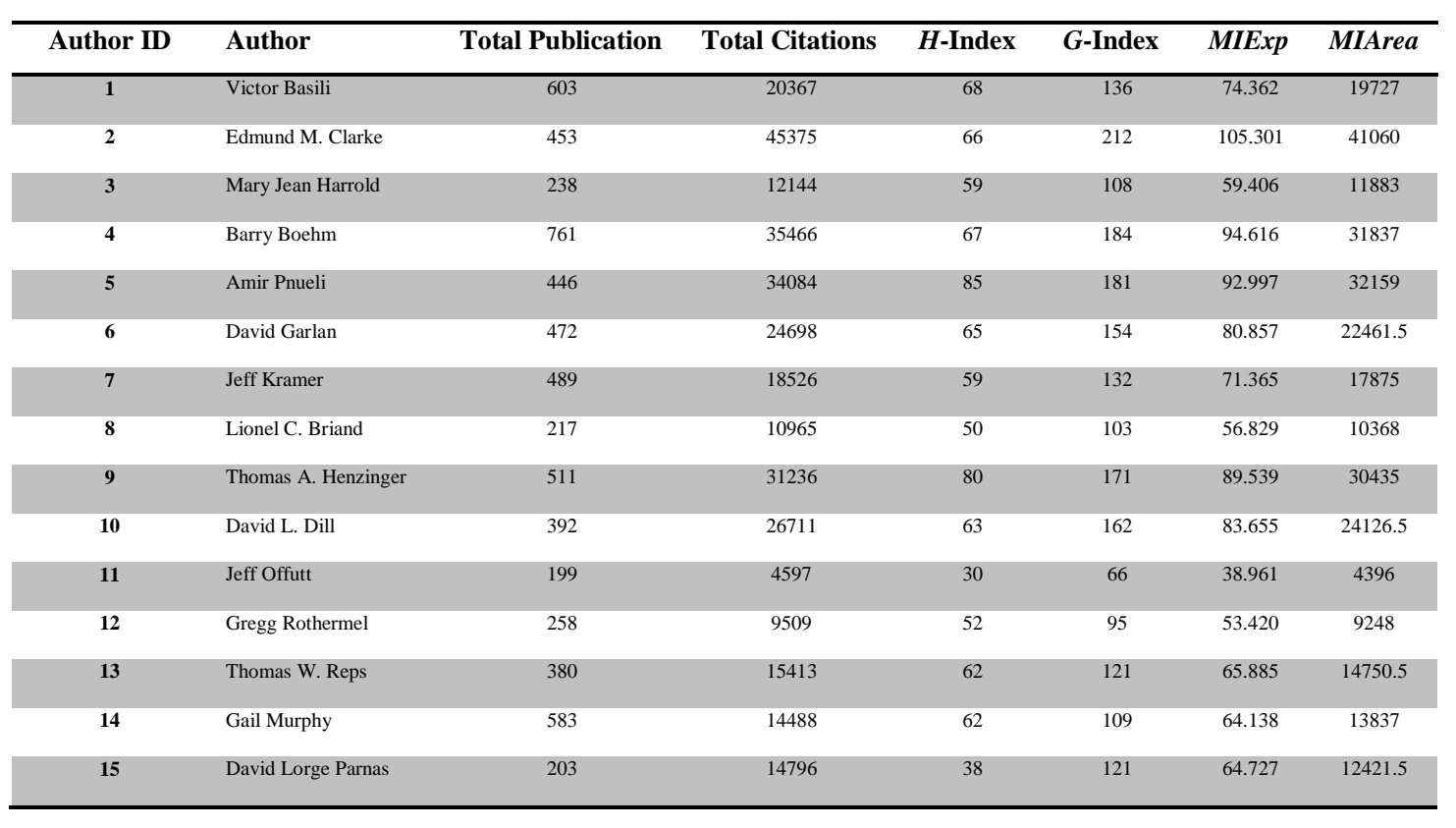

Table 11 shows the rank of each author ordered based on the indices under study. The $G$ index, MIexp and MIArea have the same point of view on authors ranking, and it is clear that they are strongly correlated. However, the sensitivity property of the proposed indices could make them more effective indicators on authors' impacts.

Table 11. Fifteen Authors Ordered according to their Index Values

\begin{tabular}{|c|c|c|c|c|c|c|c|c|c|c|c|c|c|c|c|}
\hline$\infty$ & \multicolumn{15}{|c|}{ Rank } \\
\hline 7 & 1 & 2 & 3 & 4 & 5 & 6 & 7 & 8 & 9 & 10 & 11 & 12 & 13 & 14 & 15 \\
\hline & \multicolumn{15}{|c|}{ Author ID } \\
\hline Based on $H$-Index & 5 & 9 & 1 & 4 & 2 & 6 & 10 & 13 & 14 & 3 & 7 & 12 & 8 & 15 & 11 \\
\hline Based on $G$-Index & 2 & 4 & 5 & 9 & 10 & 6 & 1 & 7 & 13 & 15 & 14 & 3 & 8 & 12 & 11 \\
\hline Based on MIExp-Index & 2 & 4 & 5 & 9 & 10 & 6 & 1 & 7 & 13 & 15 & 14 & 3 & 8 & 12 & 11 \\
\hline Based on MIArea-Index & 2 & 5 & 4 & 9 & 10 & 6 & 1 & 7 & 13 & 14 & 15 & 3 & 8 & 12 & 11 \\
\hline
\end{tabular}


There are different points of views about the production volume and its influence. It is not easy to say which scenario is the best? Those authors who have few publications and many citations, or those who have many publication and few citations? We can say that production volume is an important factor, since productive researchers tend to share their thoughts with others in the research community, even though their publications may not get a large number of citations. On the other hand, productivity can be seen as a rival for publication quality (if we consider citation number is the quality metric).

Regardless of these points of views, we showed in this work through the use of a number of artificial examples and real data that MIExp and MIArea indices can be more balanced, comprehensive and sensitive to evaluation publications if compared with other citation indices. However, the values produced by MIArea are too high and it is not easy to deal with MIArea in its current form. We proposed the idea of using Area under curve, but we should conduct more experiments in order to normalize the MIArea index values while preserving its sensitivity.

\section{Conclusions}

All citation indices, including proposed ones in this paper depend mainly and radically on the number of citations that each research paper received Currently, the $H^{-}$and $G$-indices are the most common indices used to assess the authors and their publications. However, there are many limitations related to indices' calculations. Most of those limitations are related to the sensitivity of the indices and the coverage or comprehensives of the index to cover different publication or author aspects in addition to the pure numbers. In this paper, we proposed and evaluated new indices: MIExp and MIArea which provide simple enhancements to the behaviors of: $H$ - and $G$-indices úsing the exponential function and the concept of area under curve. The evaluation proces showed that the new indices can solve the problems and behave in a more effective manner than traditional indices. More experiments and in depth studies are needed to normalize the MIAreprvalues as they are too large to deal with. On the other hand, it is critical to keep the senstivity of those indices that we showed, where each index can response to any addition in publications or citations.

\section{References}

[1] M. Franceschet "A Cluster Analysis of Scholar and Journal Bibliometric Indicators", Journal of the American Society for Information Science and Technology, vol. 60, no. 10, (2009), pp. 1950-1964.

[2] J. E. Hirsch, "An index to quantify an individual's scientific research output", Proceedings of the National Academy of Science of the USA, vol. 102, no. 46, (2005), pp. 16569-16572.

[3] L. Bornmann and H.-D. Daniel, "Does the H-index for ranking of scientists really work? Scientometrics, vol. 65, no. 3, (2005), pp. 391-392.

[4] W. Glänzel "On the H-index - a mathematical approach to a new measure of publication activity and citation impact”, Scientometrics, vol. 67, no. 2, (2006b), pp. 315-321.

[5] B. Cronin and L. Meho, "Using the H-index to rank influential information scientists", Journal of the American Society for Information Science and Technology", vol. 57, no. 9, (2006), pp. 1275-1278.

[6 Lornmann, and H.-D. Daniel, "What do we know about the h index?" Journal of the American Society for Information Science and Technology, vol. 58, (2007), pp. 1381-1385.

[7] T. Braun, W. Glänzel, and A. Schubert, "A Hirsch-type index for journals”, Scientometrics, vol. 69, no. 1, (2006), pp. 169-173.

[8] J. E. Hirsch, "Does the H-index have predictive power?", Proceedings of the National Academy of Sciences of the United States of America, vol. 104, no. 49, (2007), pp. 19193-19198.

[9] W. Glänzel, "On the opportunities and limitations of the $H$-index", Science Focus, vol. 1, (2006a), pp. 1011.

[10] B. Jin, L. Liang, R. Rousseau and L. Egghe, "The R- and ARindices: Complementing the H-index", Chinese Science Bulletin, vol. 52, no. 6, (2007), pp. 855-863.

[11] L. Egghe, "How to improve the H-index?" The Scientist, vol. 20, no. 3, (2006a), p. 14. 
[12] L. Egghe, "Theory and practise of the G-index", Scientometrics, vol. 69, no. 1, (2006b), pp. 131-152.

[13] L. Egghe, "An improvement of the H-index : The G-index. ISSINewsletter, vol. 2, (2006c), pp. 8-9.

[14] M. Schreiber, C. C. Malesios, and S. Psarakis, "Exploratory factor analysis for the Hirsch index 17 h-type variants, and some traditional bibliometric indicators", Journal of Informetrics, vol. 6, no. 3, (2012), pp. 347358.

[15] L. Bornmann, R. Mutz, S. E. Hug, and H.-D. Daniel, “A multilevelmeta-analysis of studies reporting correlations between the $\mathrm{h}$ index and 37 different $\mathrm{h}$ index variants", Journal of Informetrics, vol. 5, no. 3, (2011), pp. 346-359.

[16] SCI2S (Soft Computing and Intelligent Information Systems) - SCI2S Thematic Public Websites - H-index and Variants, (2013), Retrieved July 4, 2013,http://sci2s.ugr.es/hindex/.

[17] P. D. Batista, M. G. Campiteli, and O. Kinouchi, "Is it possible to compare researchers with different scientific interests?", Scientometrics, vol. 68, no. 1, (2006), pp. 179-189.

[18] M. Schreiber, "To share the fame in a fair way, $h_{m}$ modifies $h$ formulti-authored manuscripts", New Journal of Physics, vol. 10, (2008), pp. 040201.

[19] M. Schreiber, "Fractionalized Counting of Publications for the G-index", Journal of the American Society for Information Science and Technology, vol. 60, no. 10, (2009), pp. 2145-2150.

[20] M. Schreiber, "A case study of the modified g index: Counting multi-author publications factionally", Journal of Informetrics, vol. 4, (2010a), pp. 636-643.

[21] M. Schreiber, "How to modify the G-index for multi-authored manuscripts", Jounal of Informetrics, 4, (2010b), pp. 42-54.

[22] B. Jin, "The AR-index: complementing the H-index", ISSI Newsletter, vol,3, no 1, (2007), p. 6.

[23] M. Kosmulski, "A new Hirsch-type index saves time and works equally well as the original H-index", ISSI Newsletter, vol. 2, no. 3, (2006), pp. 4-6.

[24] Q. Wu, "The w-index: A measure to assess scientific impact by focusing on widely cited papers", Journal of the American Society for Information Science and Technology, vol. 61 no. 3, (2010), pp. 609-614.

[25] B. H. Jin, "H-index : an evaluation indicator proposed by scientist", Seience Focus, vol. 1, no. 1, (2006), pp. 8-9. (In Chinese)

[26] R. Rousseau, "New developments related to the Hirsch index" Science Focus, vol. 1, no. 4, (2006), pp. 2325.

[27] M. Maabreh, and I. Alsmadi, "A Survey of Impact and Citation Indices: Limitations and Issues", International Journal of Advanced Science and Technology, vol. 40, (2012), pp. 35-54.

[28] A. Sidiropoulos, D. Katsaros, and Y Manolopothlos "Generalized Hirsch H-index for disclosing latent facts in citation networks", Scientometrics, vol. 72 no 2, (2007), pp. 253-280.

[29] L. Bornmann, R. Mutz, and H-D. Daniel, "Are there better indices for evaluation purposes than the $h$ index? A comparison of nine different variants of the $h$ index using data from biomedicine", Journal of the American Society for In formation Science and Technology, vol. 59, no. 5, (2008), pp. 830-837.

[30] L. Egghe, and R. Rousseau, "An H-index weighted by citation impact", Information Processing and Management, vol. 44. (2008), pp. 770-780.

[31] S. Alonso, F. Cabrerizo, E. Herrera-Viedma, and F. Herrera, "hG-index: a new index to characterize the scientific output of researchers bassed on the h- and g-indices", Scientometrics, vol. 82, no. 2, (2010), pp. 391-400.

[32] F. J. Cabrerizo, S. Alonso, E. Herrera-Viedma, and F. Herrera, "q2-Index: Quantitative and qualitative evaluation based on the number and impact of papers in the Hirsch core", Journal of Informetrics, vol. 4, no. $1,(\mathbf{2 0 1 0})$, pp. 2328.

[33] F. Y. Ye and R. Rousseau, "Probing the H-core: an investigation of the tail-core ratio for rank distributions". Scientometrics, vol. 84, no. 2, (2009), pp. 431-439.

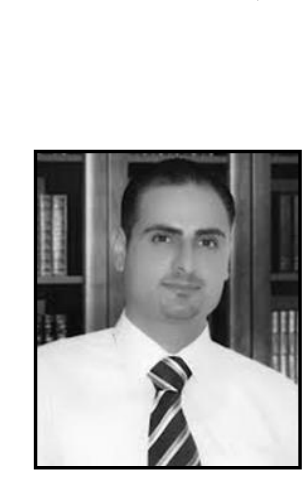

\section{Authors}

Majdi Ahmad Maabreh, he is an Oracle applications developer and DBA at Hashemite University, Jordan. He received his bachelor's degree in computer science from Yarmouk University in Jordan in July 2004. Since 2004, he joined the team of programming and development of database systems at the Hashemite University. He received his master degree in Computer Information Systems from Yarmouk University in 2012. The most topics interest includes 
Data Mining, Information Retrieval as well as Databases Technology.

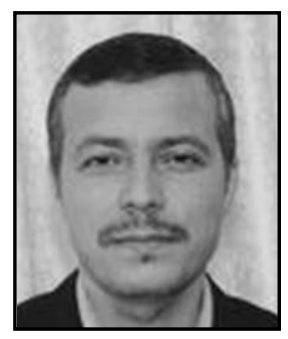

Izzat Mahmoud Alsmadi, he is an assistant professor in the department of computer information systems at Yarmouk University in Jordan. He obtained his Ph.D degree in software engineering from NDSU (USA), his second master in software engineering from NDSU (USA) and his first master in CIS from University of Phoenix (USA). He had B.sc degree in telecommunication engineering from Mutah University in Jordan. He has several published books, journals and conference articles largely in software engineering and information retrieval fields.

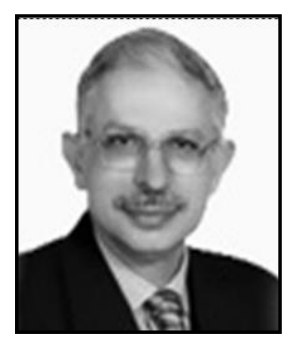

Mohammed Naji Alkabi, he obtamed his Ph $D$. degree in Mathematics from the University of Lodz/Poland (2001), his master's degree in Computer Science from the University of Baghdad/Iraq (1989), and his bachelor degree in statistics from the University of Baghdad/Iraq (1981). Monammed Naji AL-Kabi is an assistant Professor in the Faculty of Sciences and IT, at Zarqa University. Prior to joining Zarqa University, he worked many years at Yarmouk Univensity in Jordan, Nahrain University and Mustanserya University in Mrae. $\mathrm{He}$ also worked as a part time lecturer at Jodan Uniyersity of Science and Technology, and Sunderland university. AL-Kabi's research interests include Information Retrieva Web search engines, Data Mining, Sentiment analysiland opinion mining, Natural Language Processing and Software Engineering. He is the author of more than 68 peer reviewed articles in these topics. His teaching interests focus on information retrieval, Web programming, data mining, DBMS (ORACGE, MYSQL \& MS Access).
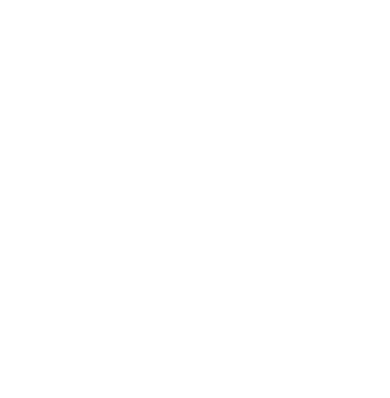

(2) 
International Journal of Hybrid Information Technology

Vol.7, No.3 (2014)

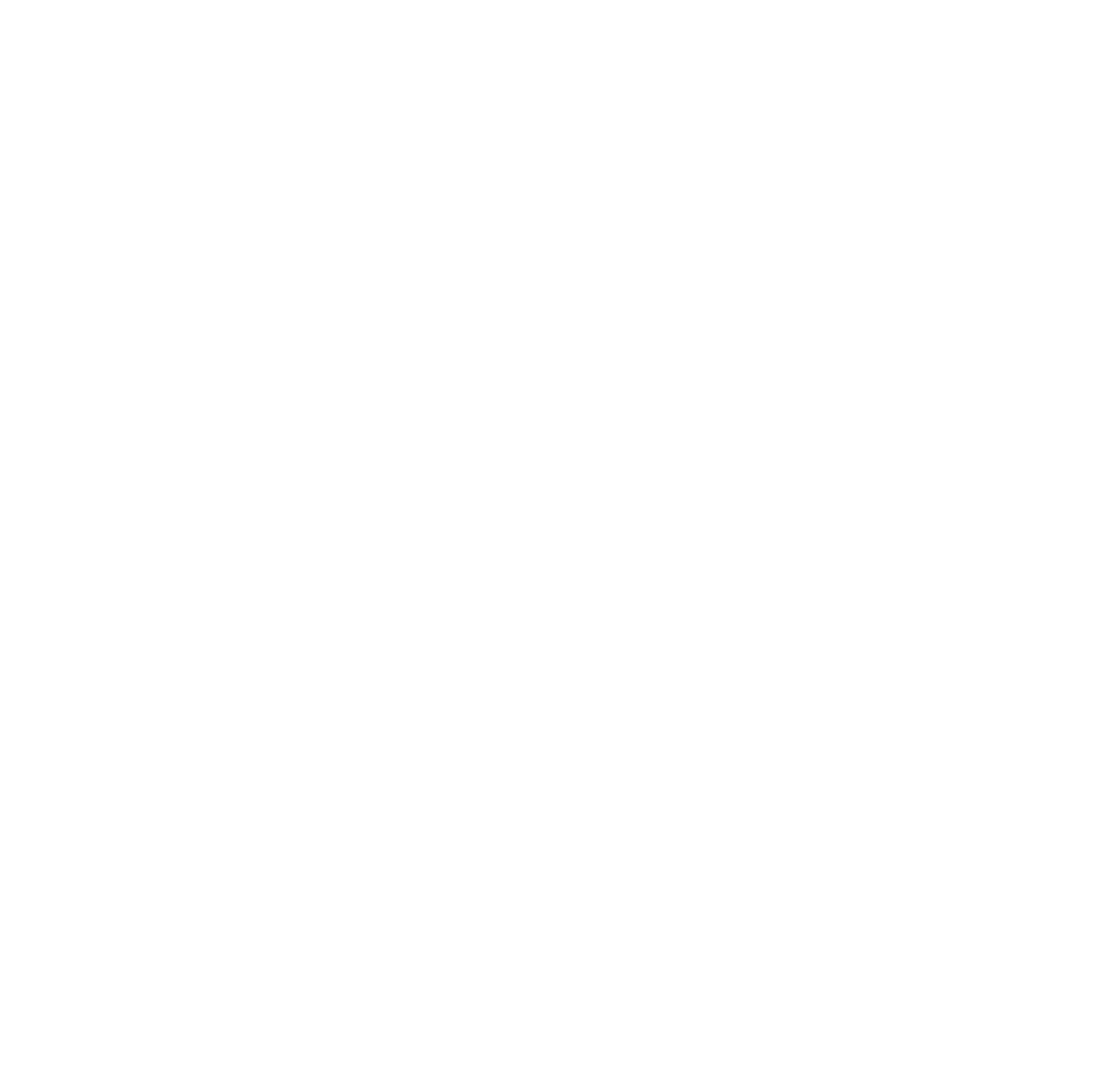

